\title{
OPTIMAL INFORMATION EXTRACTION OF LASER SCANNING DATASET BY SCALE-ADAPTIVE REDUCTION
}

\author{
Yufu Zang ${ }^{1 *}$, Bisheng Yang ${ }^{2}$ \\ ${ }^{1}$ School of Remote Sensing \& Geomatics Engineering, Nanjing University of Information Science \& Technology, Nanjing \\ 3dmapzangyufu@nuist.edu.cn \\ ${ }^{2}$ State Key Laboratory of Information Engineering in Surveying, Mapping and Remote Sensing, Wuhan University, Wuhan
}

\author{
Commission III, WG III /1
}

KEY WORDS: Multi-scale, Surface variation, Radial basis function, Just-Noticeable-Difference, Degradation

\begin{abstract}
:
3D laser technology is widely used to collocate the surface information of object. For various applications, we need to extract a good perceptual quality point cloud from the scanned points. To solve the problem, most of existing methods extract important points based on a fixed scale. However, geometric features of 3D object come from various geometric scales. We propose a multi-scale construction method based on radial basis function. For each scale, important points are extracted from the point cloud based on their importance. We apply a perception metric Just-Noticeable-Difference to measure degradation of each geometric scale. Finally, scale-adaptive optimal information extraction is realized. Experiments are undertaken to evaluate the effective of the proposed method, suggesting a reliable solution for optimal information extraction of object.
\end{abstract}

\section{INTRODUCITON}

LiDAR technology is highly valuable in many application domains such as urban planning, geography, cultural heritage protection and others because of its accuracy, speed and penetrability. However, the high density of LiDAR data leads to an enormous increase in data volume, which brings great challenges with respect to data storage, processing, display and transmission (Liu and Zhang, 2011). Therefore, it is necessary to extract the optimal information from the original points. In principle, important points should be kept and points that are not important should be reduced. However, as a result of the increasing availability of high density data, some relatively important points are also eliminated to achieve a higher reduction ratio, and some points that are not important should also be kept to preserve the geometric information of surface.

Various approaches have been proposed to tackle this issue. These algorithms can be classified into three categories: the random method, the point subtractive method and the point additive method (Chen, et.al., 2015). Immelman et.al. (2011) and Anderson et.al. (2005) applied the random extraction method. To meet the reduction ratio, a portion of points are randomly reduced. This method is simple and fast, but it does not consider the point importance so it has low accuracy. Oryspayev et.al. (2012) applied the point subtractive method, which reduces points with lower errors until a tolerance is reached. The error at each point is the distance from the point to its corresponding triangle constructed by the new local points after removing this point. This method is time consuming that it is impractical to reduce huge dataset. Different from that, Moenning et.al. (2003) proposed a point additive reduction method. Firstly, randomly take a subset from the input point cloud to establish the 3D Delaunay triangular mesh. Then define a precise distance function on this subset. Finally according to the distance from point to its corresponding triangle, add points to this subset until the error threshold or the desired number of points is reached.

To our knowledge, viewing an object (e.g., tree), we can notice geometric features of different spatial scale. For small spatial scale feature, leaf features can be noticed. And for large one, branch and tree trunk features can also be found. Geometric features of multi spatial scales form the complete information of the object. However, the above methods are performed at a unique spatial scale, which is doomed to be incomplete and misleading.

In order to derive and analyse comprehensive information of one object, multi-scale scheme is often account for this phenomenon. Arefi et.al. (2008) proposed an automatic multiscale modeling approach in three scales of detail. This method constructs the buildings which are formed by combination of flat roof, gabled roof and hipped roof segments. The examples illustrate this multi-scale representation of city buildings performs quite well. For free-form object, characteristics of curve surface are also considered in methods to retain the feature information of each scale. Pauly et.al. (2006) applied the surface diffusion equation to the discrete multi-scale surface representation. The benefit of this hierarchy is the decoupling of shape and detail at different scales. Luo et.al. (2011) proposed a multi-scale representation approach based on random walks to generate archaeological line drawings automatically instead of manual drawings. This method reduces redundant lines detected on the rough and noise surface. Additionally, some constructions of three dimensional scale space (Mokhtarian et.al., 2001; Schlattmann et.al., 2006) replace two dimensional pixel densities with three dimensional coordinates directly. These approaches produce erroneous results since the extrinsic geometry of the original data was modified. To solve the above problems, Bariya et.al. (2012) put forward a registration and recognition method based on scale-dependent features. In this method, normal vector map was used instead of the original 
range image. The Intrinsic Geometric Scale of feature points was used to construct scale-dependent features. However, the target of this method is range image. For a three dimensional object, many projection angles should be considered. Similarly, Novatnack et.al., (2007) and Hua et.al., (2008) unwrapped the surface of model onto a two dimensional image. The scale space was constructed based on the geometric attributes retained on the image. However, when a surface patch containing complex features is squeezed into a very small domain, problems will appear.

In this paper, we propose a multi-scale extraction method to describe geometric features of different spatial scales. The small scale points describe the small features while large scale points focus on the features of larger spatial scale and ignore the small ones. If the geometric scale selected is smaller, data redundancies may exist; if the geometric scale selected is larger, many meaningful information may be lost. To extract optimal information of point cloud, we measure the visual degradation of point cloud of each scale. Unnecessary and unnoticeable geometric features are reduced as much as possible to provide a good quality data for further applications.

The rest of the paper is structured as follows: Section 2 and Section 3 describes our proposed method to construct the geometric multi-scales based on radial basis function model, and select optimal scale based on a perceptual metric. We report the outcomes of a number of experiments undertaken to demonstrate the validities of the proposed method in Section 4. Finally, in Section 5 we draw our conclusions.

\section{SURFACE VARIATION AND IMPORTANCE MEASUREMENT}

\subsection{Surface Variation}

Apply PCA and local covariance statistics to estimate the normal vector of each point based on its neighbourhood points (Nurunnabi et al., 2015). The calculated normal vectors of two adjacent points may have opposite directions. To ensure the accuracy of surface variation, consistent propagation of normal vectors is realized by traversing minimum cost spanning tree (König et al., 2009). For a local area, calculate the cosine value between normal vectors of current point and one neighbour by:

$$
C_{i}=1-\vec{n}_{0} \cdot \vec{n}_{i}
$$

The upper value is between 0 and 2. The surface variation of current point is evaluated by the mean error of neighbourhood cosine values, as:

$$
S_{0}=\frac{\sum_{i=1}^{n} \mid C_{i}-\text { mean } \mid}{n}
$$

Here $\mathrm{n}$ is the number of neighbours, mean is the mean value of neighbourhood $C$ values. This value describes the surface variation since the value is larger when local surface has larger variation.

\subsection{Importance Measurement}

The key to construct multi-scales is to determine whether one point is important at a given scale. In this section, we propose an effective important metric based on radial basis function. This function considers the influence of neighbourhood point distribution on the current point, it is a successful tool for the approximation. The function is assumed to have the form:

$$
s(p)=\sum_{j=1}^{N} \lambda_{j} \varphi\left(\left\|p-p_{j}\right\|\right)+c(p), c \in S^{d}
$$

Where $\|\cdot\|$ is the Euclidean norm on $\mathrm{R}^{\mathrm{d}}, c(p)$ is the polynomial part and $S^{d}$ denotes the linear space containing polynomials in d variables. Many classical choices for radial basis functions have been proposed (e.g. Gaussian, multi-quadric and cubic). To reduce the calculation complexity of RBF, Wendland (1995) put forward unified formula of basic function, defined as:

$$
\varphi(r)= \begin{cases}(1-r)^{q} p(r), & 0 \leq r \leq 1 \\ 0, & 1<r\end{cases}
$$

Various forms of $\varphi(r)$ with continuity of $\mathrm{C}^{0}, \mathrm{C}^{2}, \mathrm{C}^{4}$ was given. Here $\mathrm{C}^{2}$ continuity: $\varphi(r)=(1-r)_{+}^{4}(4 r+1)$ is applied popularly. If the distance from one neighbour to current point is smaller than support radius $\mathrm{r}$, then $\varphi(r)=0$. This local supporting property reduce the calculation complexity.

We evaluate the importance of one point by two aspects: surface variation and the distribution of neighbourhood points. The Importance metric is defined as:

$$
\left\{\begin{array}{l}
I_{0}=S_{0} \exp \left(-w \sum_{i=1}^{N} m_{i} \varphi\left(\left\|P_{i}-P_{0}\right\| / \lambda\right)\right. \\
m_{i}=\frac{S_{i}}{\sum_{j=1}^{N} S_{j}}
\end{array}\right.
$$

Here $S_{0}$ is the surface variation of current point $P_{0}, \lambda$ is the support radius factor, $P_{i}$ is one point from the valid supporting region, $w$ is the weight coefficient, deciding the degree of influence of neighbours on current point, $m_{i}$ is the proportion of neighbourhood surface variation. In the formula (5), RBF is used to measure the contribution of neighbourhood point to current point. This contribution depends on the number and spatial distribution of neighbour points. When the neighbour points distribute densely, the contribution is large, importance will decrease, vice versa. This characteristic of the formula suggest that the influence of neighbourhood distribution can be described well. 


\section{MULTI-SCALE CONSTRUCITON AND OPTIMAL SELECTION}

\subsection{Edge Points Detection}

Surface variation outlier values will appear in the edge areas, we apply an angle criterion method to detect the edge points. The spatial distribution characteristics of local points is used to detect the edge points. If the neighbours of one point distribute on one side, this point is regarded as edge point; if the neighbours distribute around this point uniformly, it is regarded as internal point, as shown in Figure 1.

The specific procedures are: Firstly, local tangent planes are constructed by one point and its neighbours, and project neighbours to this plane to get a $2 \mathrm{D}$ point set; Then calculate angles formed by two consecutive projected neighbours to the current point, and sort all the angles in decreased order; Finally, get the largest gap to judge whether it is edge point.

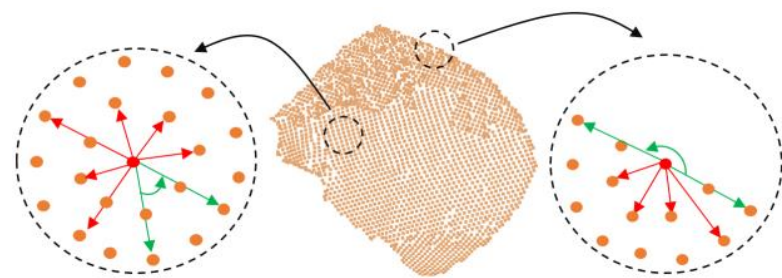

Figure 1. Neighbourhood distribution of one inner point and one edge point

\subsection{Extraction of multi-scale Points}

Based on importance metric and the detected edge points, we apply the following procedures to extract points of one scale: a. Suppose the Final Point Set of current scale is empty. Surface variation of each point is calculated based on the neighbourhood size of current scale.

b. Sort original points by their surface variation values in decreasing order, and select the variation value of the point at $90 \%$ as the Importance threshold.

c. Take one point (non-edge point) from the sorted point set successively to the Final Point Set. Calculate the Importance of this point based on the new neighbours in Final Point Set by Formula 5. If I value is larger than threshold, keep it, or remove it from Final Point Set.

d. Repeat step c until all the points of original point set is considered. Take the Final Point Set together with the smaller scales as current scale points.

\subsection{Optimal Scale Selection}

For a surface, removing a point will generate a stimulus to human vision. To measure the perceptual impact, JustNoticeable-Difference (JND) is used (Xu et al., 2014). However, the application of JND is limited since it needs complex skeleton extraction. In this section, we improve JND measurement by replacing the skeleton with $3 \mathrm{D}$ smoothed surface.

When a point is removed, its local geometric information is represented by its closest remaining point. We use $\Delta \rho$ to describe the change when point $\mathrm{A}$ is removed from the mesh (as show in Figure 2), point B is the nearest remaining point of A. $\rho$ is defined as the perception distance from the closest remaining point to the smoothed surface. The stimulus variation is defined as:

$$
K=\frac{\Delta \rho_{A B}}{\rho}=\frac{\left|r_{A}-r_{B}\right|}{r_{B}}
$$

Here $r_{A}$ and $r_{B}$ are the distances from point $\mathrm{A}$ and $\mathrm{B}$ to the smoothed surface. $\mathrm{K}$ is the JND threshold. According to the Formula, removing point $\mathrm{A}$ has less stimulus than removing point C. Due to Webber's equation, the stimulus value less than $\mathrm{K}$ is not perceptible to human vision. The degradation will be recorded if the stimulus is larger than K. In this paper, experiments suggest $\mathrm{K}=0.8$.

To generate the 3D smoothed surface, we extend the 2D Gaussian kernel. The 3D Gaussian kernel is defined as:

$$
G(i, j ; \delta)=\frac{1}{2 \pi \delta^{2}} \exp \left[-\frac{1}{2 \delta^{2}} d_{i j}{ }^{2}\right]
$$

Here $\delta$ is the mean square root of Gaussian kernel function, $d_{i j}$ is the distance from neighbourhood point $j$ to current point $i$. This process is repeated for each point, and the new points define the smoothed surface. Similar to 2D smoothing, the larger mean square root is, the larger geometric features will be smoothed out. Larger mean square root is selected in this paper.

Based on JND model, we apply salient information distortion metric (Shi et al., 2010) to measure the degradation of each scale. According to the degradation value, we select the optimal scale with least points to reserve the most perceptual information.

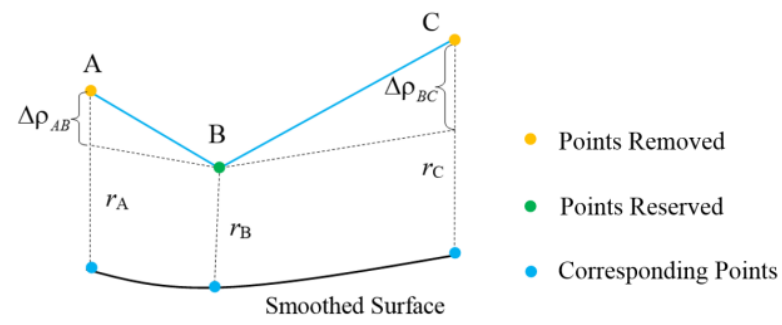

Figure 2. Stimulus variations generated by removing points

\section{EXPERIMENTAL RESULTS AND ANALYSIS}

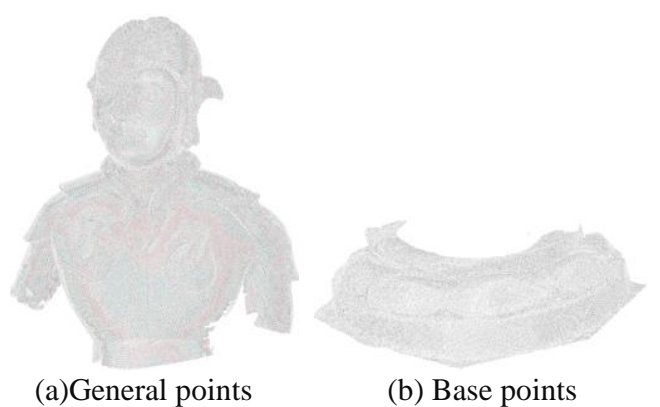

Figure 3. Experimental point clouds.

To evaluate the efficency of proposed method, General and Base are selected for experiment. They are scanned by Handyscan 3D scanner with the average span of point clouds about $1.0 \mathrm{~mm}$ and $0.2 \mathrm{~mm}$, respectively. Both of them have multi-scale of geometric details and rich redundancies. 


\subsection{Detection of Edge Points}
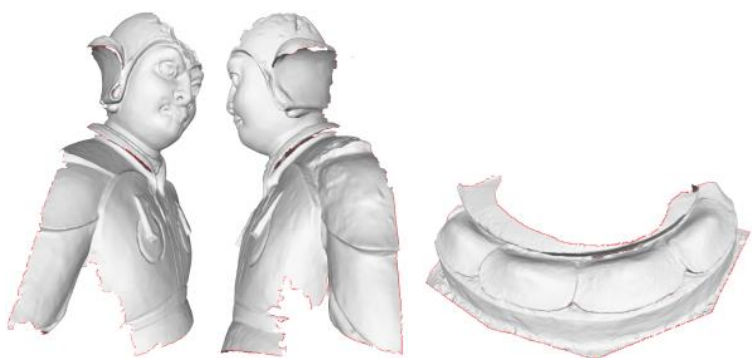

Figure 4. Edge points detected

Some outlier edge points may be introduced by surface variation. They will affect the construction of multi-scale. From Figure 4 we can see that the edge points of two point clouds can be detected effectively (red dots indicate the detected edge points). This insures the validity of extraction of multi-scale points.

\subsection{Extraction of Multi-scale Points}

Extracted points of scale 3,4,5,6 are shown in Figure 5. It suggests that important points of different scales can be well reserved with a suitable point span; And in smooth areas, local maximum feature points can also be reserved so that no obvious holes can be found. In addition, points of different scales reflecting different scale features can well represent the geometric information of object.

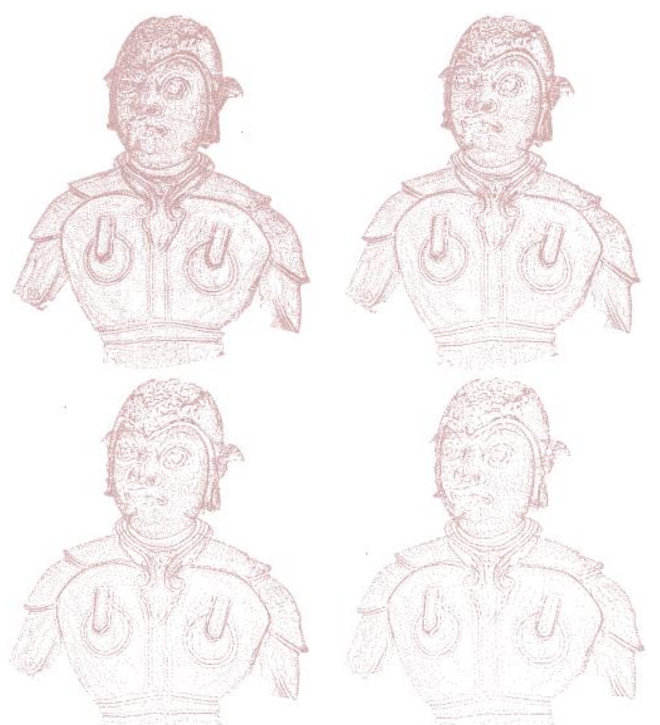

(a) General Points of Scale 3,4,5,6
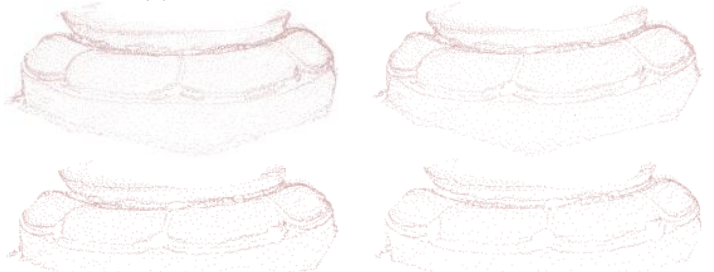

(b) Base points of Scale 3,4,5,6

Figure 5. Multi-scale of points extracted

\subsection{Optimal Scale Selection}

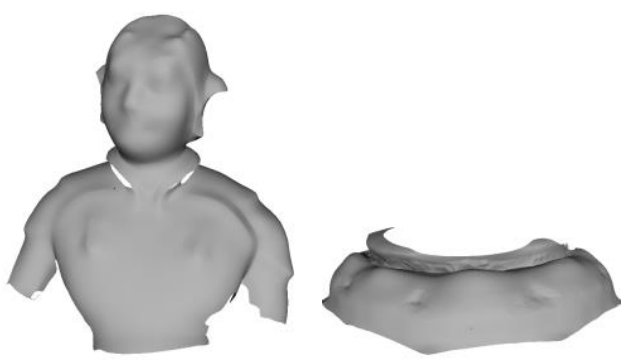

Figure 6. Smoothed surface of two datasets

The smoothed surfaces of two point clouds are shown in Figure 6 . It suggests that the smoothed surfaces are basically the base planes of objects, and it is beneficial to use the smoothed surface instead of skeleton.

Based on the stimulus value in Formula 6, we calculate SIDM value of each scale to measure its perceptual degradation. In Figure 7, there is a step change in SIDM values of multi-scales (e.g., between scale 4 and scale 5). This suggests that the stimulus variations caused by deleting small scale features (e.g., scale 2, 3, 4) are not enough be noticeable for human vision. This is in accord with the visual characteristics. The objective of the paper is to extract least points to describe the most perceptual information. Hence we select scale 4 of General and Base datasets as the optimal scales.

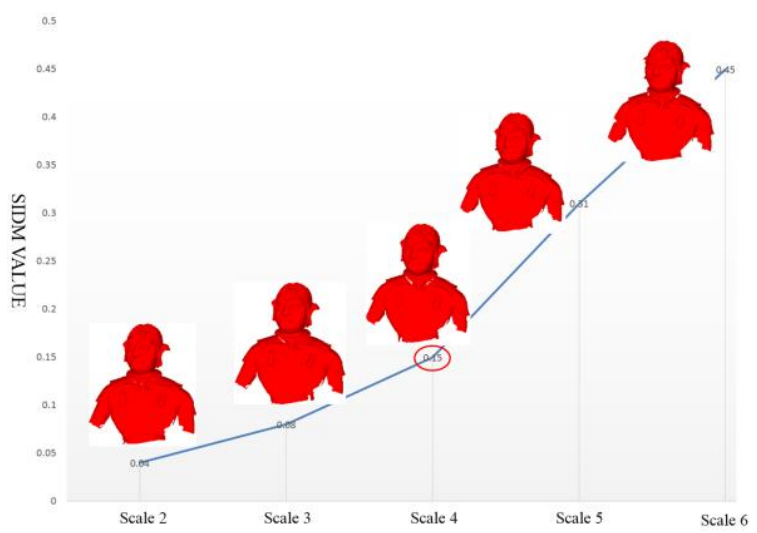

(a) General data

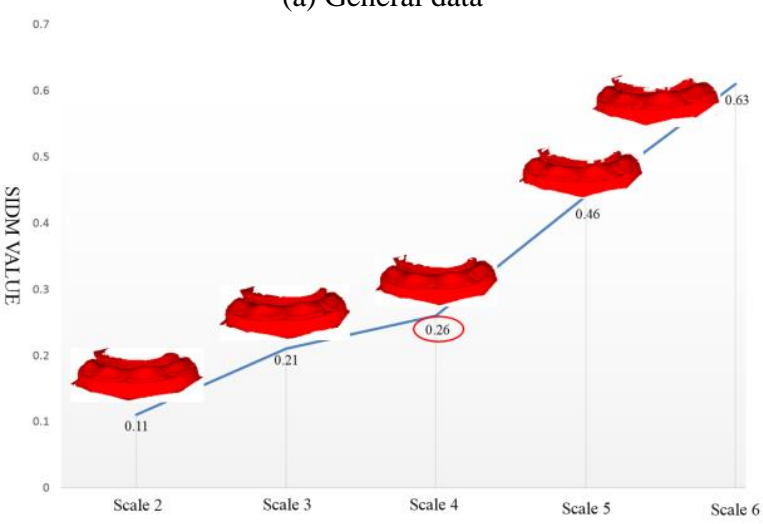

(b) Base data

Figure 7. Optimal scale selection

\section{CONCLUSIONS}

Optimal information extraction of 3D point clouds is an inevitable process in various applications. This paper proposes 
an effective method to extract optimal points from point cloud based on scale-adaptive reduction. The method introduces an importance metric by combining the surface variation and radial basis function. And then detect the edge outliers and extract points of multi-scale. Lastly, we propose a new stimulus variation measurement to evaluate degeneration of each scale for optimal selection, leading to a good perceptual quality.

\section{ACKNOWLEDGEMENTS}

Work described in this paper is supported by National Science Foundation of China project under Grant No. 41701529, and University Science Project of Jiangsu Province under Grant No. 17KJB420004, and Project for Introducing Talent of NUIST under Grant No. 2016r062, and National Science Foundation of China project under Grant No. 41371431.

\section{REFERENCES}

Liu, X., Zhang, Z., 2011. Effects of LiDAR Data Reduction and Breaklines on the Accuracy of Digital Elevation Model. Survey Review43.323:614-628(15).

Chen, C., Yan, C., Cao, X., Guo, J., Dai, H., 2015. A greedybased multi-quadric method for LiDAR-derived ground data reduction. ISPRS Journal of Photogrammetry \& Remote Sensing: $110-121$.

Immelman, J., Scheepers, L.G.C., 2011. The effects of data reduction on LiDAR-based digital elevation models. Image and Signal Processing (CISP), 2011 4th International Congress on. Vol. 3. IEEE.

Anderson, E.S., Thompson, J.A., Austin, R.E., 2005. LIDAR density and linear interpolator effects on elevation estimates. International Journal of Remote Sensing 26.18: 38893900 .

Oryspayev, D., Sugumaran, R., DeGroote, J., 2012. LiDAR data reduction using vertex decimation and processing with GPGPU and multicore CPU technology. Computers \& Geosciences 43: 118-125.

Moenning, C., Dodgson, N.A., 2003. A New Point Cloud Simplification Algorithm. In Proceedings 3rd IASTED Conference on Visualization, Imaging and Image Processing: 1027--1033.

Arefi. H., Engels, J., Hahn, M., 2008. Levels of detail in 3d building reconstruction from lidar data. IISPRS Congress Beijing Proceedings of Commission III.

Pauly, M., Leif. P., Kobbelt., Markus, G., 2006. Point-based multi-scale surface representation. ACM Transactions on Graphics (TOG) 25.2: 177-193.

Luo, T., Renju L., Hongbin Z., 2011. 3D line drawing for archaeological illustration. International Journal of Computer Vision 94.1: 23-35.

Mokhtarian, F., Nasser K., Peter Y., 2001. Curvature computation on free-form 3-D meshes at multiple scales. Computer Vision and Image Understanding 83.2: 118139.
Schlattmann, M., 2006. Intrinsic features on surfaces. Central European seminar on computer graphics. Vol. 72.

Bariya, P., Novatnack, J., Schwartz, G., 2012. 3D geometric scale variability in range images: Features and descriptors. International journal of computer vision, 99(2): 232-255.

Novatnack, J., Nishino, K., 2007. Scale-dependent 3D geometric features. Computer Vision. ICCV 2007. IEEE 11th International Conference on. IEEE: 1-8.

Hua, J., Lai, Z., Dong, M., Gu, X., Qin, H., 2008. Geodesic distance-weighted shape vector image diffusion. IEEE Transactions on Visualization and Computer Graphics, 14(6). 